\title{
Curie Extension Score
}

National Cancer Institute

\section{Source}

National Cancer Institute. Curie Extension Score. NCI Thesaurus. Code C133467.

A score assigned to a Curie body segment to indicate the quantity of metastases. 